\title{
Specific cytoarchitectureal changes in hippocampal subareas in daDREAM mice
}

Britt Mellström ${ }^{1,2+}$, Asta Kastanauskaite ${ }^{1,3,4 \dagger}$, Shira Knafo 3,9, Paz Gonzalez ${ }^{1,2}$, Xose M. Dopazo 1,2, Ana Ruiz-Nuño ${ }^{5}$, John G. R. Jefferys ${ }^{5}$, Min Zhuo ${ }^{6,7}$, Tim V. P. Bliss $^{8}$, Jose R. Naranjo ${ }^{1,2^{*}}$ and Javier DeFelipe ${ }^{1,3,4}$

\begin{abstract}
Background: Transcriptional repressor DREAM (downstream regulatory element antagonist modulator) is a $\mathrm{Ca}^{2}$ ${ }^{+}$-binding protein that regulates $\mathrm{Ca}^{2+}$ homeostasis through gene regulation and protein-protein interactions. It has been shown that a dominant active form (daDREAM) is implicated in learning-related synaptic plasticity such as LTP and LTD in the hippocampus. Neuronal spines are reported to play important roles in plasticity and memory. However, the possible role of DREAM in spine plasticity has not been reported.

Results: Here we show that potentiating DREAM activity, by overexpressing daDREAM, reduced dendritic basal arborization and spine density in CA1 pyramidal neurons and increased spine density in dendrites in dentate gyrus granule cells. These microanatomical changes are accompanied by significant modifications in the expression of specific genes encoding the cytoskeletal proteins Arc, Formin 1 and Gelsolin in daDREAM hippocampus.
\end{abstract}

Conclusions: Our results strongly suggest that DREAM plays an important role in structural plasticity in the hippocampus.

Keywords: Calcium, Arc, Dendritic trees, Spines

\section{Background}

Change in intracellular free calcium concentration has long been recognized as a universal signal underlying neuronal plasticity and adaptive responses in the CNS to different environmental stimuli $[1,2]$. Diverse signaling pathways participate in these responses, among them a specific set of proteins that decode the calcium signal in accordance with frequency, subcellular location and intensity [3-5]. Despite extensive investigation, however, a detailed mechanistic description of $\mathrm{Ca}^{2+}$-dependent signaling in the expression of the late, transcriptiondependent component of LTP and LTD remains elusive [reviewed in 6]. It was proposed that the concentration of intracellular free calcium affects dendritic spine (for simplicity, spine) density by controlling spine growth and pruning [7] and that the formation of new spines

\footnotetext{
* Correspondence: naranjo@cnb.csic.es

${ }^{\dagger}$ Equal contributors

${ }^{1}$ Spanish Network for Biomedical Research in Neurodegenerative Diseases, CIBERNED, Madrid, Spain

${ }^{2}$ National Biotechnology Center. CSIC, Darwin, 3. E-28049, Madrid, Spain Full list of author information is available at the end of the article
}

requires calcium-dependent CREB phosphorylation and CRE-dependent transcription [8].

DREAM belongs to a group of four highly conserved genes $\left(K^{+}\right.$channel interacting proteins, KChIP-1 to 4) [9, $10]$, that regulates synaptic activity through different mechanisms (Table 1) including binding to DRE regulatory sites in target genes and to other nucleoproteins like CREB [11, reviewed in 12]. Despite its potential, the role of the $\mathrm{Ca}^{2+}$-dependent transcriptional repressor DREAM in spine growth and remodeling associated with the expression of LTP and LTD has not been analyzed.

Mutation of the EF-hands in DREAM results in a $\mathrm{Ca}^{2}$ ${ }^{+}$-insensitive repressor that in vitro shuts down DREand CRE-dependent transcription in the presence of elevated intracellular levels of free $\mathrm{Ca}^{2+}[9,13]$. Mutation of a leucine-charged residue rich domain (LCD) at the Nterminal of DREAM $(\mathrm{L} 47,52 \mathrm{~V})$ prevents the interaction with CREB [13] and in combination with the EF-hand mutation generates a calcium insensitive double mutant daDREAM that specifically blocks $\mathrm{Ca}^{2+}$-/DREAMdependent transcription without blocking CREBdependent gene expression. Use of transgenic mice over expressing the $\mathrm{Ca}^{2+}$-insensitive DREAM mutant 
Table 1 DREAM is a multifunctional regulatory protein

\begin{tabular}{|c|c|c|c|}
\hline Modified function & Regulated by & Molecular mechanism & References \\
\hline $\begin{array}{l}\text { Kv4 channel gating \& } \\
\text { membrane localization }\end{array}$ & $\begin{array}{l}\text { CalciumLipids } \\
\text { Phosphorylation by GRK2 }\end{array}$ & Protein-protein interaction & $\begin{array}{l}\text { An et al., } 2000 \text { [10] Holmqvist et al., } 2001 \text { [70] } \\
\text { Ruiz-Gomez et al., } 2007 \text { [71] }\end{array}$ \\
\hline $\mathrm{Ca}^{2+}$ release from the ER & Not known & Protein-protein interaction & Lilliehook et al., 2002 [72] \\
\hline \multirow[t]{2}{*}{$\begin{array}{l}\text { Voltage-gated } \mathrm{Ca}^{2+} \text { channel } \\
\text { expression \& gating }\end{array}$} & \multirow[t]{2}{*}{ Calcium } & Transcriptional regulation & $\begin{array}{l}\text { Ronkainen et al., } 2011 \text { [73] } \\
\text { Naranjo \& Mellstrom, } 2012 \text { [24] }\end{array}$ \\
\hline & & Protein-protein interaction & Thomsen et al., 2009 [74] Anderson et al., 2010 [75] \\
\hline $\begin{array}{l}\mathrm{Ca}^{2+} \text { influx through } \\
\text { NMDA receptors }\end{array}$ & Calcium & Protein-protein interaction & Wu et al., 2010 [14] Zhang et al., 2010 [15] \\
\hline GABAergic inhibition & Calcium & Transcriptional regulation & Mellstrom et al., 2014 [16] \\
\hline $\begin{array}{l}\text { Chronic pain } \\
\text { desensitization }\end{array}$ & Calcium \& BDNF & Transcriptional regulation & Rivera-Arconada et al., 2010 [30] \\
\hline ATF6 processing & Calcium & Protein-proteininteraction & Naranjo et al., 2016 [25] \\
\hline \multirow[t]{2}{*}{ Pain perception } & \multirow[t]{2}{*}{ Calcium } & Transcriptional regulation & Carrion et al., 1999 [9] Cheng et al., 2002 [26] \\
\hline & & Protein-protein interaction & Hu et al., 2006 [76] \\
\hline
\end{tabular}

daDREAM revealed that long-term depression (LTD), a form of synaptic plasticity, was significantly impaired in daDREAM transgenic mice [14]. Moreover, contextual fear and spatial memory as well as behavioral anxiety were significantly impaired in daDREAM mice. A postsynaptic modulation of the NMDA receptor by DREAM through a $\mathrm{Ca}^{2+}$-dependent interaction with PSD-95 [14] or by the interaction with the NMDA-R1 subunit [15] could also contribute to this phenotype. In addition, expression of daDREAM in the forebrain resulted in a complex phenotype characterized by loss of recurrent inhibition and enhanced LTP in the dentate gyrus (DG), impaired learning and memory and profound changes in the expression of specific activity-dependent transcription factors in the hippocampus, including Npas4, Nr4a1, Mef2c, JunB and c-Fos [16].

Here, we report specific changes in dendritic arborization and spine density in CA1 pyramidal neurons and granule cells of the DG, respectively, in adult transgenic mice expressing the daDREAM mutant. Moreover, changes in the expression of genes related to the cytoskeleton that could participate in the modified cyto-architecture were found in daDREAM transgenic hippocampus.

\section{Results}

Individual hippocampal neurons were injected with $\mathrm{Lu}$ cifer Yellow in fixed coronal slices. We could thus readily visualize the dendritic arbor, including fine branches as well as dendritic spines, of individual neurons (Fig. 1a-c). Since in a $200 \mu \mathrm{m}$ slice the entire dendritic tree could not always be included, due to its large extension, the values regarding the total dendritic lengths and the Sholl analysis for the dendritic tree (length and number of intersections) represent the dendritic tree included in the slice. Nevertheless, as all neurons were injected at the same depth into the slice $(30 \mu \mathrm{m}$ from the surface) it is assumed that the portion of the dendritic tree included in the slice is equal in different neurons and across genotypes.

\section{Changes in the CA1 dendritic trees in daDREAM mice}

Changes in synaptic plasticity and learning and memory are associated with dendritic development $[7,17,18]$ as well as with the $\mathrm{Ca}^{2+}$-dependent growth and pruning of dendritic spines [19]. Thus, we examined whether neuronal morphology and spine density were altered in daDREAM CA1 pyramidal neurons, those that could be responsible for the modified LTD in daDREAM mice (Fig. 1b and c). Representative tracings of CA1 pyramidal neurons from wild type and daDREAM mice are shown in Fig. 1d.

Sholl analysis was used to calculate the number of dendrite crossings (intersections) and the dendritic lengths at increasing distances $(10 \mu \mathrm{m}$ interval) from soma as objective measurements of the dendritic complexity. The analysis revealed, both for apical (Fig. 2a, b) and basal (Fig. 2c and d) dendrites, a statistically significant reduction for these parameters in transgenic mice. Thus, the total dendritic tree is shorter (Fig. 2e and $\mathrm{f}$ ) and the dendritic complexity is smaller in daDREAM CA1 neurons compared to wild type.

Spine density in CA1 neurons was analyzed at increasing distances from soma (Sholl analysis) and for each branch order in apical (Fig. 3a and b) and basal (Fig. 3c and $\mathrm{d}$ ) dendrites. The result showed no difference in spine density between wt and daDREAM neurons in apical dendrites (Fig. 3a and b). In basal dendrites, however, we found a significant lower spine density in daDREAM neurons (Fig. 3c and d).

In addition we calculated the total number of spines by combining spine density and Sholl analysis (density of 


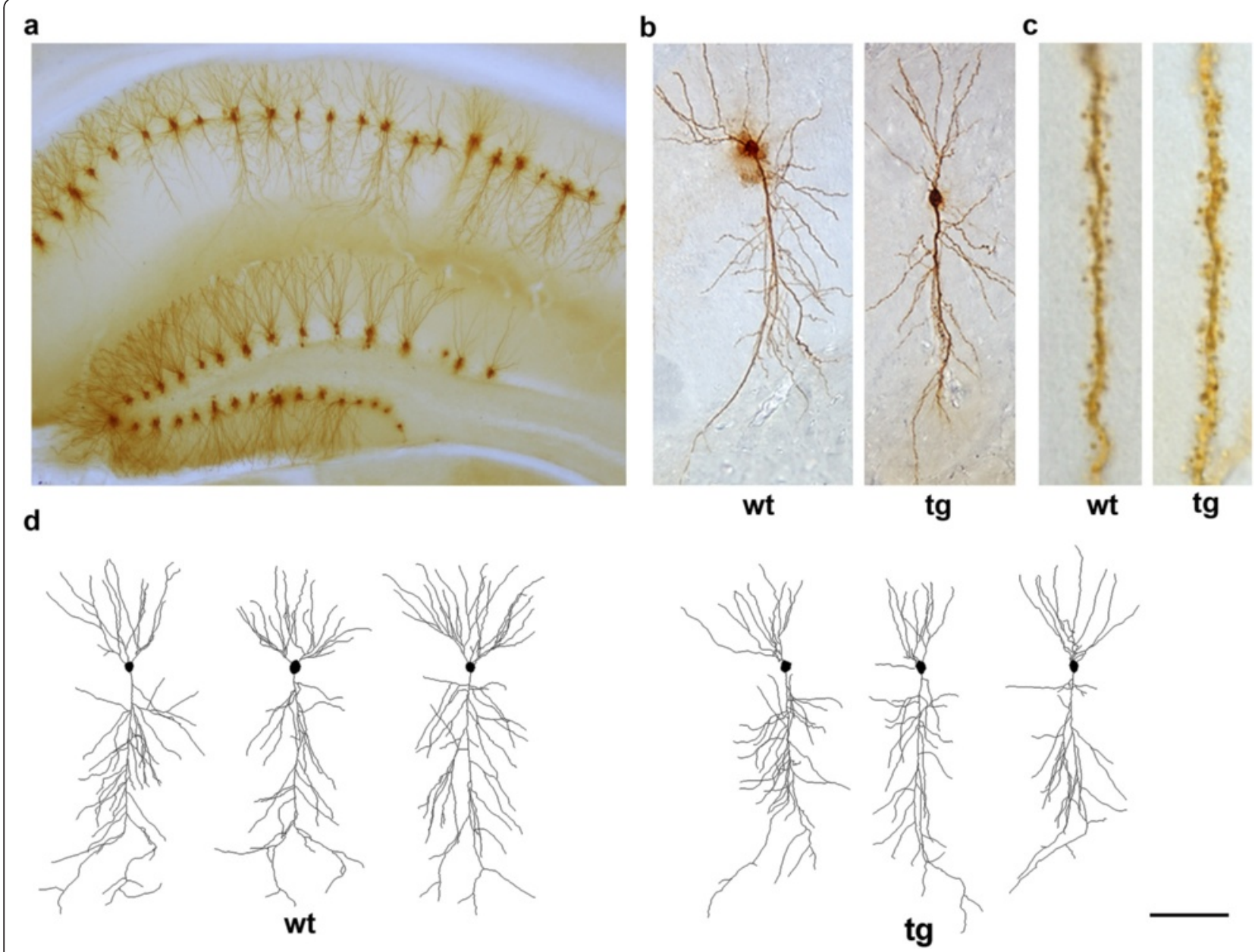

Fig. 1 Lucifer Yellow injected neurons in the hippocampus. a Panoramic view of the hippocampus showing Lucifer Yellow injected neurons in CA1 and DG areas. b Representative individual CA1 pyramidal neurons, wild type (wt) and daDREAM (tg). c High magnification photomicrographs of representative dendrites of the CA1 basal dendritic tree, showing the dendritic spines in wt and tg neurons. $\mathbf{d}$ Neurolucida reconstructions of CA1 wt and tg neurons. Scale bar, A $200 \mu \mathrm{m}, \mathrm{B} 40 \mu \mathrm{m}, \mathrm{C} 8 \mu \mathrm{m}, \mathrm{D} 50 \mu \mathrm{m}$

spines $\mathrm{x}$ dendritic length). We found a significant reduction of spines in the basal arbor of transgenic neurons (Fig. 3e).

\section{Changes in spine density in DG granular neurons of daDREAM mice}

Individual granular neurons were injected with Lucifer Yellow and traced in three-dimensions (Fig. 1a and Fig. 4a and b). Representative tracings of DG granular neurons from wild type and daDREAM mice are shown in Fig. 4c.

As for CA1 pyramidal neurons, Sholl analysis was used to calculate the number of dendrite crossings (intersections) and the dendritic lengths at increasing distances (10 $\mu \mathrm{m}$ interval) from soma as objective measurements of dendritic complexity. This analysis revealed for daDREAM granule neurons no significant differences in the dendritic length (Fig. 5a and b) and in the number of intersections at any distance from the soma (Fig. 5c).

Spine density, however, was significantly higher in daDREAM compared to wild type granule neurons as measured by Sholl analysis (Fig. 5d) and per branch order (Fig. 5e). As a result, the total number of spines calculated by combining spine density and Sholl analysis (density of spines $x$ dendritic length) was significantly increased in DG transgenic granule cells (Fig. 5f).

\section{Expression of cytoskeletal related genes are modified in daDREAM hippocampus}

To relate morphological changes in transgenic hippocampus with potential differences in gene expression in daDREAM mice, we search for modified levels of mRNAs encoding proteins related to actin polymerization and cytoskeleton, some of which have been related to calcium homeostasis, synaptic plasticity and learning and memory, 


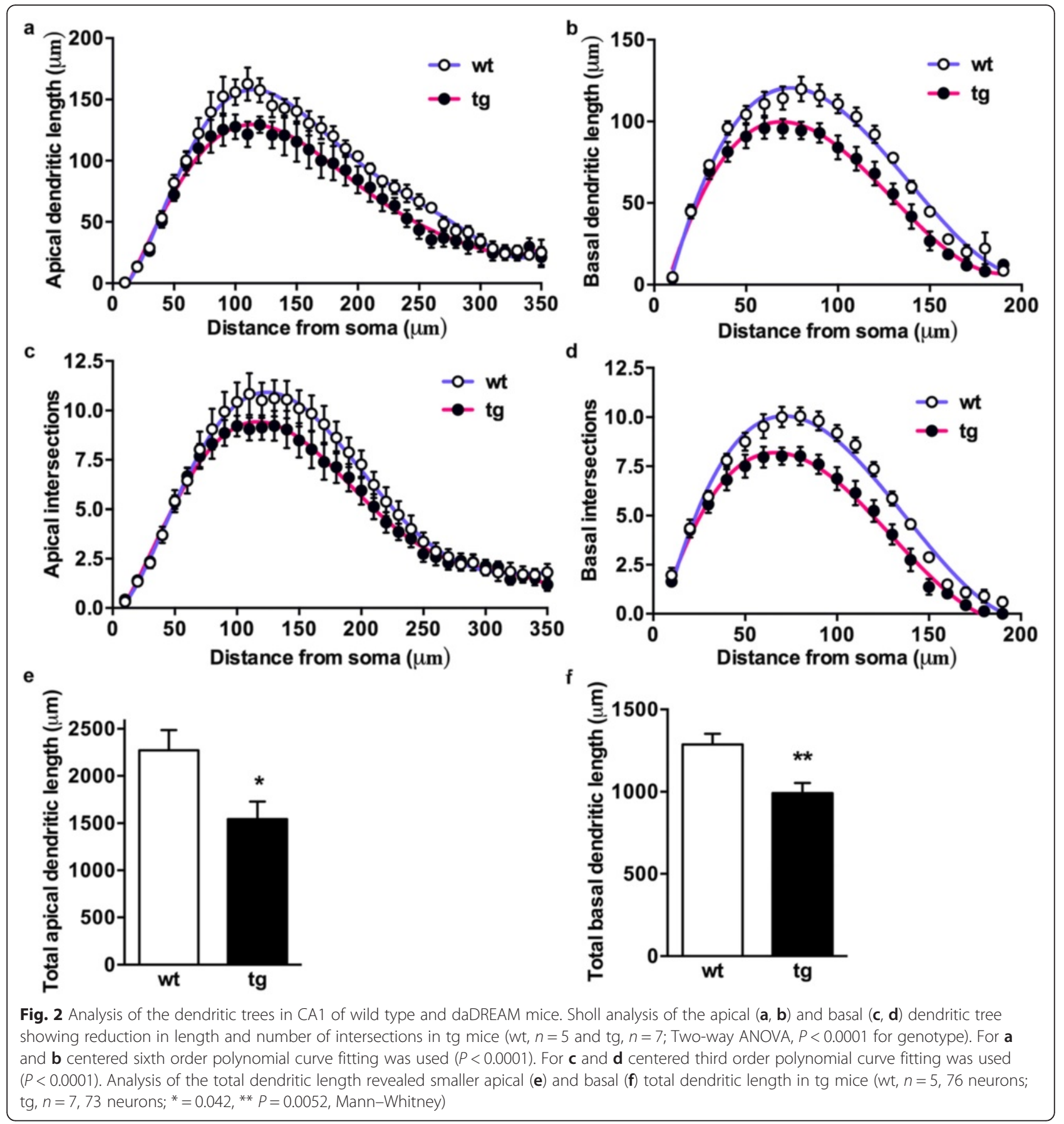

i.e. Arc [20-22] and Gelsolin [23]. For this, we revisited the results from a genome-wide analysis of daDREAM hippocampus (Gene Expression Omnibus accession number GSE17844) [16] and validated specific messengers by quantitative real-time PCR. Expression levels for Arc, Fhod3, Tmod3 and formin 1 were reduced, while a significant increase in gelsolin mRNA was observed in daDREAM hippocampus (Fig. 6a and b). These changes were specific since no significant alteration was observed for others, including formin2, spire1 and cap1 (Additional file 1). Notably, expression of Arc and gelsolin was not significantly modified in DREAM ${ }^{-/-}$hippocampus, a lack of effect likely due to compensation by other KChIPs expressed in this brain area (Fig. 6a).

Reduced expression of Arc in whole hippocampus was observed also at the protein level in daDREAM mice (Fig. 6c). Furthermore, analysis of Arc protein in hippocampal subareas showed a decrease in Arc content in the CA1 and the DG from transgenic mice compared to wild-type (Fig. 6c). 


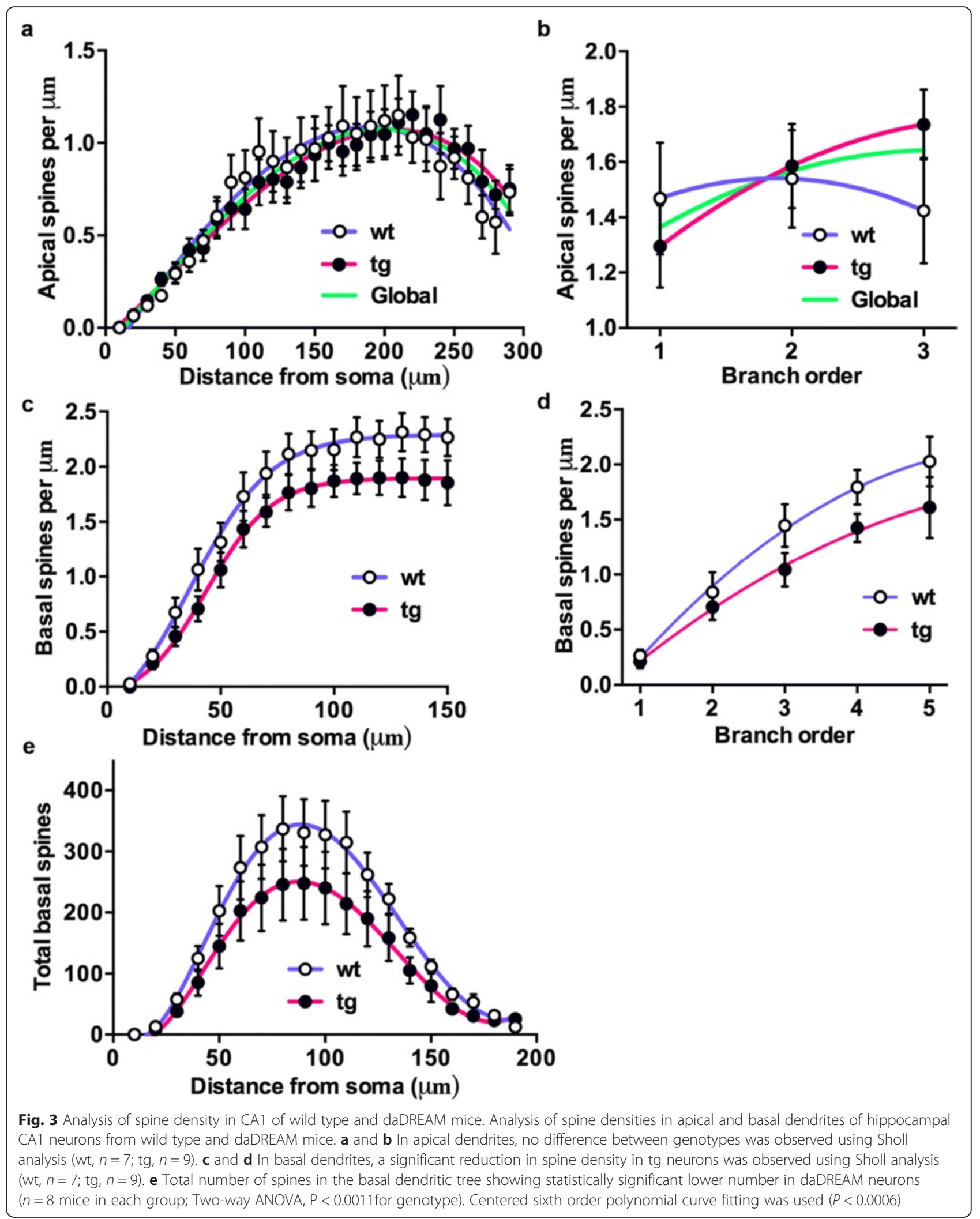




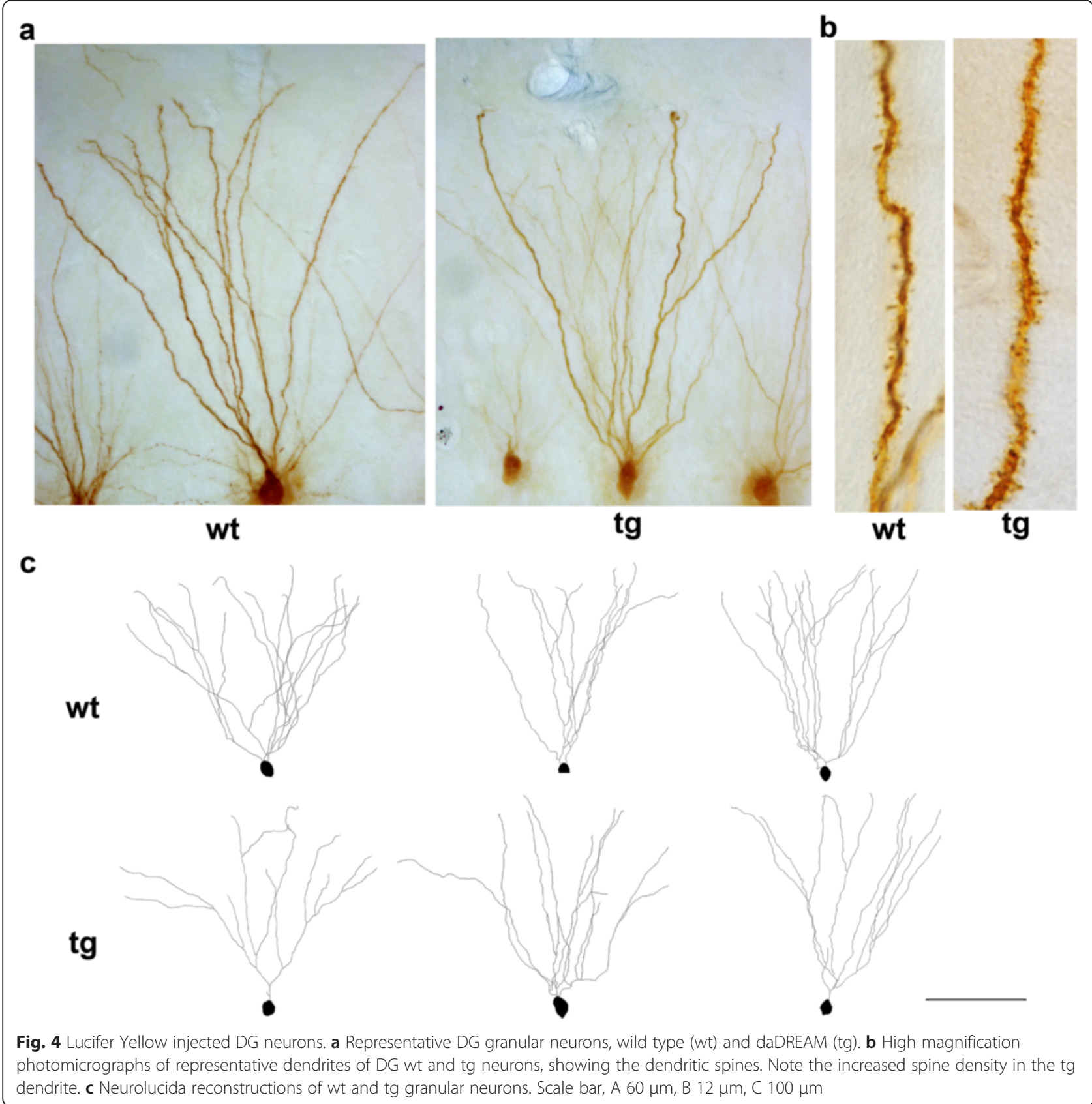

Taken together, these results indicate that daDREAMinduced changes in the expression levels of genes related to actin polymerization and to the cytoskeleton, may underlie changes in neuronal morphology and connectivity and could be related to the alterations in more elaborated functions as learning and memory [16].

\section{Discussion}

DREAM regulates the expression of several genes, which are important for $\mathrm{Ca}^{2+}$ and protein homeostasis and synaptic plasticity $[24,25]$. This includes activity-dependent expression of c-fos and Npas4 $[9,16]$, as well as, effector genes directly responsible for synaptic events and calcium homeostasis in the postsynaptic neuron such as prodynorphin, BDNF and the sodium-calcium exchanger 3 [26-29]. In addition, expression of daDREAM in the CNS has been associated with modified LTD and LTP responses and a severe impairment in learning and memory formation $[14,16,30]$.

Here, we present evidence for permanent changes in the microanatomy of CA1 pyramidal cells and granule cells of DG, which suggest alterations of hippocampal 


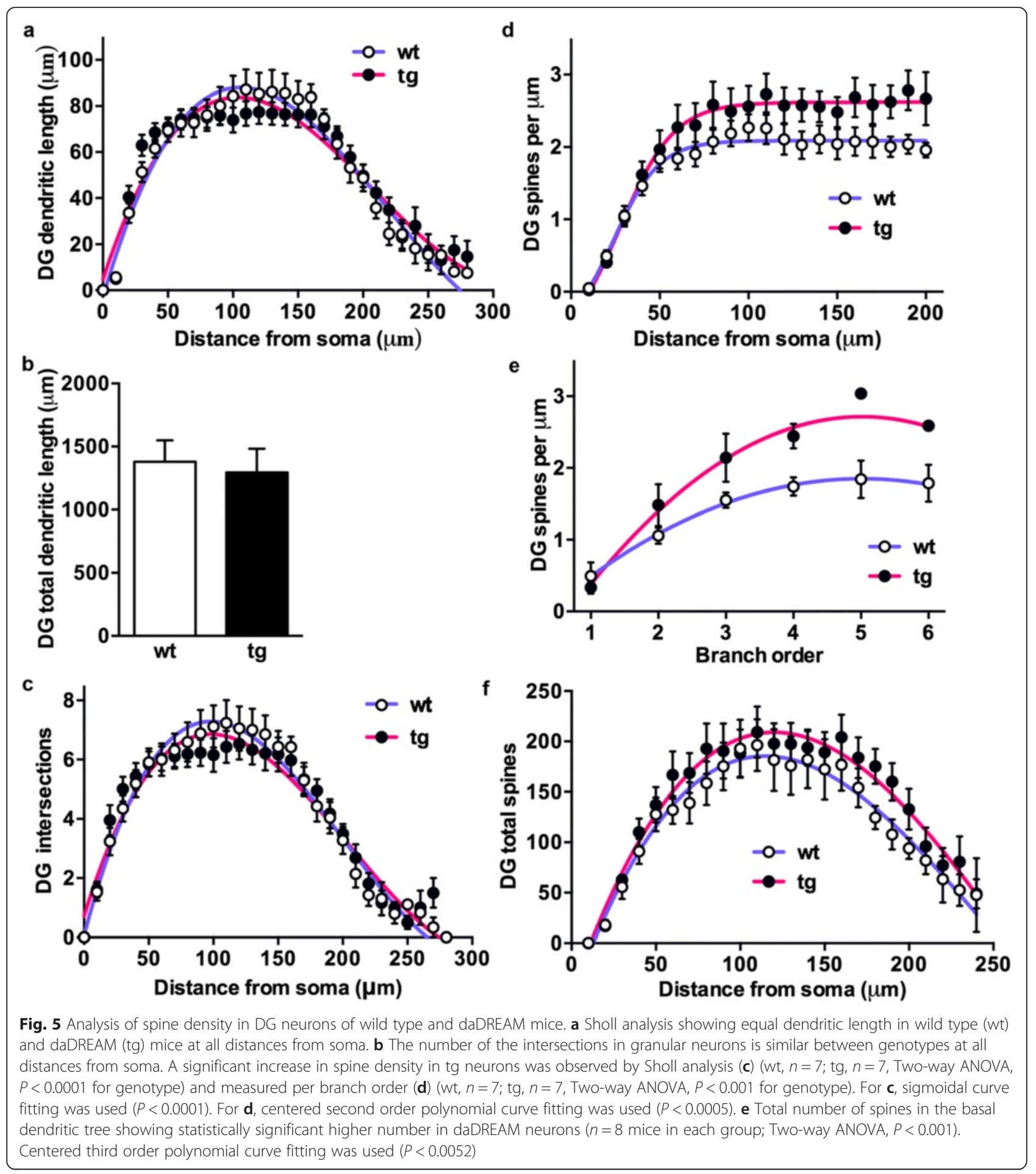

connectivity, as well as in the expression of cytoskeletal genes, Arc, formin1 and gelsolin, which could underlie altered LTD and LTP in daDREAM mice.

Modified protein expression of Arc, Formin1 and Gelsolin has been associated with changes in actin polymerization, spine density, dendritic growth and impairment in long-term forms of synaptic plasticity, including LTP and LTD [22, 31, 32]. Thus, it is well characterized that decreased protein expression of Arc impairs LTD in CA1 neurons [33] and in Purkinje cells [20] and that reduced LTD depends on a decrease in the translation rate of pre-existing levels of $\operatorname{Arc}$ mRNA in CA1 neurons [34]. Moreover, knockdown of Arc in basolateral amygdala impairs long-term extinction of 


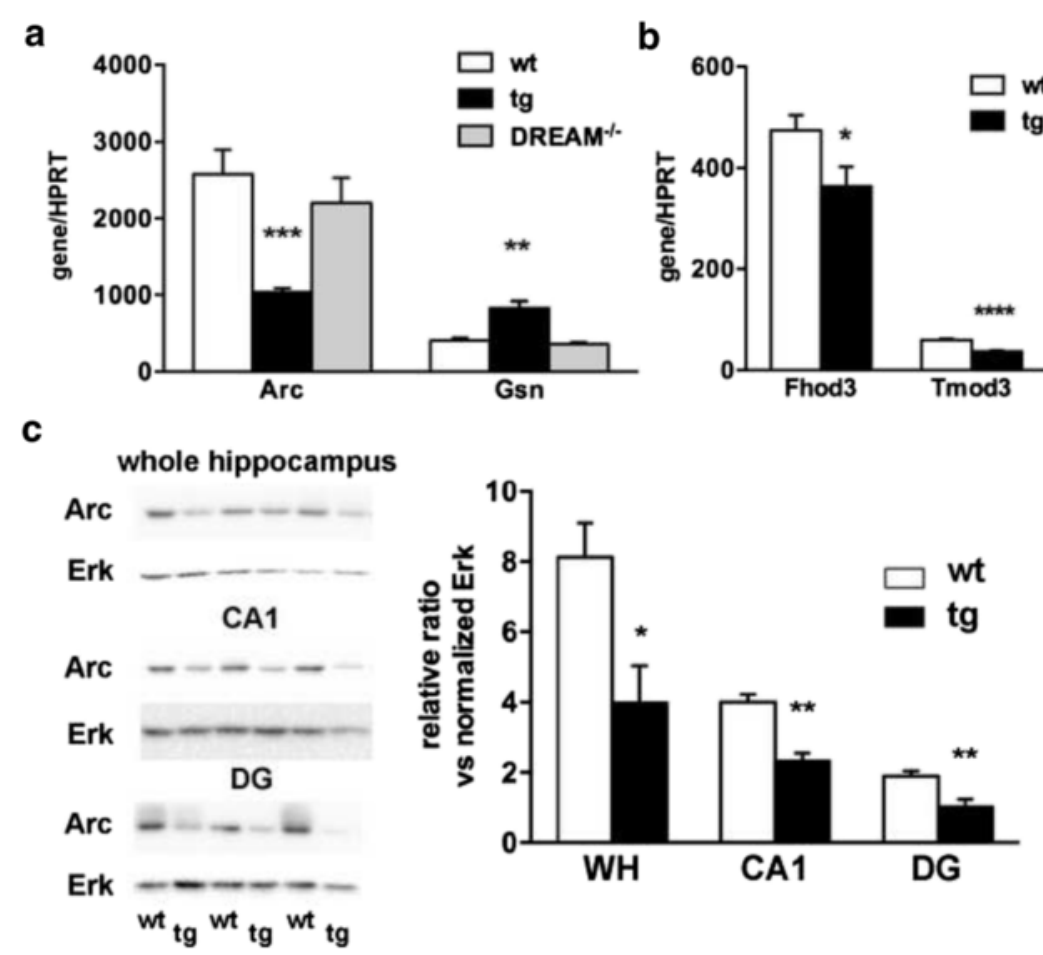

Fig. 6 Changes in the expression of cytoskeletal related genes in daDREAM hippocampus. a, b Quantitative real-time PCR analysis of the indicated genes in hippocampus from wild type (wt), daDREAM ( $\mathrm{tg}$ ) and DREAM knockout (DREAM ${ }^{-1-}$ ) mice. Values are normalized with respect to HPRT mRNA content. Results are the mean \pm SEM. In (a), ${ }^{* *} P<0.01,{ }^{* * *} P<0.001$ (One-way ANOVA followed by Dunnett's multiple comparison, $n=10$ ). In (b), ${ }^{*} P<0.05,{ }^{* * * *} P<0.0001$ (Student's $t$-test, $\left.n=17\right)$. c Western blot analysis of the Arc protein in whole hippocampus (WH), CA1 and DG from wild type (wt) and daDREAM mice (tg). Five mice were analyzed in each group. Representative gels are shown. ${ }^{*} P<0.05,{ }^{* *} P<0.01$ (Mann Whitney)

fear memory [35] and total ablation of Arc in $\mathrm{Arc}^{-1-}$ mice results in decreased spine density and altered spine morphology in CA1 and DG neurons [36] while no change in spine density was reported earlier [33].

Our results in daDREAM mice add new pieces of information to this functional scenario. We found no difference in spine density between wild type and daDREAM CA1 neurons in apical dendrites, while in basal dendrites we found a significant lower spine density in daDREAM neurons. This difference could be relevant since the mechanisms of induction and maintenance of LTP differ in apical (stratum radiatum) and basal dendrites (stratum oriens) of hippocampal CA1 pyramidal neurons [37-41]. Furthermore, we found an increase in spine density in the granule cells of the DG that may account for the increase in LTP in daDREAM mice observed in this region. Reduced expression of Arc mRNA in whole daDREAM hippocampus translates to a reduction of Arc protein in whole hippocampus, that is also observed in CA1 and DG hippocampal subareas. Thus, complex changes in hippocampal cytoarchitecture in daDREAM mice are not explained solely by the reduction in Arc protein content and might be understood only in the context of a largely modified transcriptional scenario due to daDREAM overexpression [16]. Future experiments should explore changes in the level and distribution of other cytoscheletal proteins, changes in the fine morphology and functionality of the spines in daDREAM hippocampal neurons and the relationship of these two events with the different mechanisms of induction and maintenance of LTP between apical and basal dendrites.

Decreased formin1 expression and reduced dendritic complexity in daDREAM CA1 neurons may well be related. It has been reported that Formin1 mediates Neurogenin3-induced dendritogenesis and synaptogenesis in cultured hippocampal neurons [42]. Moreover, the increase in gelsolin expression in daDREAM hippocampus may be involved in the increase in spine density in daDREAM granule cells in the DG, since a role in the stabilization of actin polymerization has been proposed for Gelsolin [31]. Nonetheless, expression of cap1 a regulator of Cofilin, an actin related protein that participates in spine changes during LTD [43], is not modified in daDREAM neurons. Thus, long term structural changes and changes in the expression of cytoskeletal proteins have a relatively good correlation that is not complete, suggesting the involvement of additional 
mechanisms and/or the occurrence of specific changes within hippocampal regions that should be further explored to fully understand the modified long term synaptic plasticity in daDREAM mice.

Down regulation of Arc and formin 1 gene expression is in line with the intrinsic repressor activity of DREAM, while the induction of gelsolin expression may be secondary to primary changes in target genes or the consequence of the interaction between DREAM and other nucleoproteins important for gelsolin gene regulation. Whether DREAM acts directly on the Arc and formin 1 promoters or indirectly affecting the transactivating effect of SRF on the distal enhancer that directs basal and activity-dependent transcription of the $\operatorname{Arc}$ gene [44-46] remains to be clarified. In addition, the decrease in $A r c$ expression could be secondary to the repression of Npas4 and BDNF expression in daDREAM hippocampus [16]. Nevertheless, it has been shown that Forminmediated actin polymerization is involved in the activation of SRF in the nucleus and that SRF activation results in changes of the expression levels of multiple cytoskeletal-related proteins [47-49]. Whether the down regulation of the formin 1 gene is the primary transcriptional event in daDREAM neurons and whether changes in the expression of Fhod3, Tmod3 and gelsolin are related to changes in SRF function are questions that remain to be investigated.

A large dendritic arbor implies that a wider region of the cortex must be sampled, while a more complex branching pattern may determine the degree to which the integration of inputs is compartmentalized within their arbors [50-54]. Greater potential for compartmentalization results in a significant increase in the representational power and a greater capacity for learning and memory [54, reviewed in 55]. In addition, each spine receives at least one excitatory synapse and such spines represent the main target of these synapses [56]. Thus, the differences in the size, branching pattern and spine density of cells result in variation in the total number of putative excitatory inputs sampled by each cell and in the integrative properties of these neurons. The lower number of spines in basal dendrites of CA1 and increased spine density in transgenic granule cells of the DG suggests decreased connectivity in the CA1 and enhanced connectivity in the DG. Nevertheless, we have not analyzed the different types of spines present in excess in daDREAM versus wild type neurons, and more important, we do not know how synapses are modified during LTD and LTP in transgenic hippocampus.

Studies using DREAM knockout mice showed no difference with wild type mice in paired pulse facilitation, resting membrane potential or input-output relation of fEPSPs [57]. Moreover, DREAM deficient mice did not show an obvious phenotype in a place-learning version of the Morris water maze test [26] and had only a slight hormonal dependent changes in memory in fear conditioning tests [58] and slight increase in LTP in the dentate gyrus of the hippocampal formation [57]. In the latter study, enhanced LTP in the DG of DREAM knockout mice could be mimicked by potassium channel blockers and was associated with decreased A-type current density [57]. Therefore, it was concluded that $\mathrm{Kv} 4$ potassium channels are important for mediating the function of DREAM in synaptic plasticity. However, work in transgenic spinal cord neurons have shown no change in the activity of Kv4 channels in daDREAM transgenic neurons indicating that the lack of spinal sensitization in transgenic mice is not related to change in $\mathrm{K}^{+}$currents but rather related to the transcriptional control of BDNF by mutated DREAM [30]. In the same way, no significant change in the basal expression of Arc, forming 1 and gelsolin were observed in this study in the hippocampus of DREAM -/- mice. Absence of strong phenotypes in DREAM/KChIP3 deficient mice is likely due to the functional redundancy among DREAM/ KChIP proteins and their overlapping expression patterns $[27,59]$, as occurs after genetic ablation of KChIP2 [60] or KChIP1 [61], in which compensation by other KChIP proteins also results in mild phenotypes.

Activity-dependent synaptic plasticity might transform small and silent synapses to larger and fully functional synapses. Expression of the calcium insensitive DREAM mutant in daDREAM mice indicates that not only basal but also activity-dependent changes in gene expression are reduced in daDREAM neurons. Thus, functional synaptic changes upon membrane depolarization may not occur or be reduced in daDREAM compared with wild type neurons. Future studies should address these different possibilities.

\section{Conclusions}

Our results strongly suggest that DREAM plays an important role in learning-related structural plasticity in the hippocampus.

\section{Methods}

\section{Transgenic mice}

The generation of transgenic mice has been reported previously [29, 30]. This study was performed in daDREAM mice line 26 , specific details about this transgenic line can be found in [16].

All experimental protocols involving the use of animals were performed in accordance with recommendations for the proper care and use of laboratory animals and were performed under authorization through the regulations and policies governing the care and use of laboratory animals (EU directive $n^{\circ} 86 / 609$ and Council of Europe Convention ETS123, EU decree 2001-486 and 
Statement of Compliance with Standards for Use of Laboratory Animals by Foreign Institutions $n^{\circ}$ A5388-01, National Institutes of Health (USA).

\section{Morphometric analysis}

Mice were anesthetized with pentobarbital $(0.04 \mathrm{mg} / \mathrm{kg})$ and transcardially perfused with saline followed by $80 \mathrm{ml}$ of $4 \%$ paraformaldehyde made in $0.1 \mathrm{M}$ phosphate buffer $(\mathrm{pH}$ 7.4). The brains were removed from the skull, and postfixed in the same solution for $24 \mathrm{~h}$. Coronal sections $(150 \mu \mathrm{m})$, were cut with the aid of a vibratome, and prelabeled with $10^{-5} \mathrm{M}$ 4,6-diamidino-2-phenylindole (DAPI, Sigma D9542).

Cell injection methodology has been described in detail elsewhere [62-64]. Briefly, cells in the posterior third of the left dorsal hippocampus (anteroposterior -2.10 to $-2.70 \mathrm{~mm}$ from bregma [65] were injected individually with $4 \%$ Lucifer Yellow ( $\mathrm{CH}$, Aldrich) in $1 \mathrm{M} \mathrm{LiCl}$ (pH 7.4) [66], by passing steady hyperpolarizing current through the electrode $(\mathrm{C}-0.5$ to $-1.0 \mathrm{nA})$. Current was applied until the distal tips of each neuron fluoresced brightly.

Following injection, the sections were processed with an antibody to Lucifer Yellow (1:400,000 in stock solution [2 \% bovine serum albumin (Sigma A3425), $1 \%$ Triton $\mathrm{X}-100(\mathrm{BDH} \quad 30$ 632), $5 \%$ sucrose in $0.1 \mathrm{M}$ phosphate buffer]), followed by a biotinylated species specific secondary antibody (Amersham RPN 1004; 1:200 in stock solution) and a biotin-horseradish peroxidase complex (Amersham RPN1051; 1:200 in 0.1 M phosphate buffer). DAB (3,3'-diaminobenzidine; Sigma $D$ 8001) was used as the chromogen. The slides were coded prior to the morphological analysis. The code was not broken until after the quantitative analysis was completed.

For the analysis of dendrite morphometry, neurons were only included in the analysis if they had clearly distinguishable dendritic trees and all dendrites were completely filled. In order to determine whether the dendritic arbor structure is altered in daDREAM mice, neurons were traced with the aid of a computerized data collection system, Neurolucida (Neurolucida V6; MicroBrightfield, Inc., Williston, VT) coupled to an Olympus microscope (BX51), using a 40x objective (NA 0.8) with examination, as needed, at 100x (Oil, NA 1.35). The Sholl analysis [67], which calculates the number of dendritic bifurcations and length at $10 \mu \mathrm{m}$-interval distance points starting from the soma, was automatically performed with Neuroexplorer 4.50 program (MicroBrightfield, Inc., Williston, VT). Total dendritic length was also generated.

For the analysis of spine density of granular neurons in the DG, one dendrite with its branches was traced and the dendritic spines were marked. All types of spines were included in the spine counts, and no correction factors were applied to the spine counts, as dendrite tracing at high power allows the visualization of all spines that issue from the dendrites, i.e. the DAB reaction product is more transparent than the Golgi reaction product. The reconstructed data were exported to Neurolucida Explorer (MicroBrightField Inc., Williston, VT) for quantitative analysis. Sholl analysis [67] was applied to determine the spine density at increasing distances from the soma.

For the statistical analysis, spine density was calculated by dividing the number of spines on a segment by the length of the segment and was expressed as the number of spines per $1 \mu \mathrm{m}$ of dendrite. Densities of spines on dendrites were averaged for a cell mean, and the neurons from each animal were averaged for an animal mean. The total number of spines in the basal dendritic tree of the pyramidal cells in CA1 was calculated by multiplying the mean number of spines of a given portion of dendrite by the mean number of branches for the corresponding region and animal, over the entire dendritic tree (obtained by Sholl analysis) [68]. Normality was tested using the Kolmogorov-Smirnov test. For Sholl analysis, longitudinal distributions were first assessed using 2-way ANOVA considering all interactions, and then, modeled using non-linear regression model fitting to various curves. The best-fitted curves were compared to obtain difference between genotypes. The significance criterion was set at $P<0.05$. All data shown are presented as mean \pm SEM.

\section{Quantitative real-time PCR}

RNA was isolated from hippocampal tissues using TRIzol (InVitrogene), treated with DNAse (Ambion) and reverse transcribed using hexamer primers and Moloney murine leukemia virus reverse transcriptase. To confirm the absence of genomic DNA, each sample was processed in parallel without reverse transcriptase. Quantitative real-time PCR was performed using assays from Applied Biosystems (Additional file 2). The results were normalized by quantification of HPRT mRNA using the specific primers; forward 5'-TTGGATACAGGCCAG ACTTTGTT-3' and reverse 5'-CTGAAGTACTCATT ATAGT CAAGGGCATA-3', and the probe FAM-5'TTGAAATTCCAGACAAGTTT-3'-MGB.

\section{Western blot analysis}

Mouse hippocampi were quickly removed and one was processed as whole hippocampus and from the other, hippocampal subareas CA1 and DG were dissected as described [69]. Hippocampal tissue was homogenized on ice in NETN buffer (Tris pH $8.050 \mathrm{mM}$, NaCl $250 \mathrm{mM}$, EDTA $5 \mathrm{mM}$, NP40 $0.5 \%$, supplemented with protease inhibitors, Complete Roche). Extracts were cleared by 
centrifugation (14,000 g, $20 \mathrm{~min})$. Samples (15 $\mu \mathrm{g})$ were analyzed by SDS-PAGE and immunoblot. PVDF membranes were incubated with anti-Arc (C-7, Santa Cruz) and as loading control, with anti-Erk2 (C-14, Santa Cruz). Secondary antibodies used were HRP-conjugated donkey anti-rabbit or -mouse IgG (Jackson; $1 \mathrm{~h}$, room temperature), and signals were detected with ECL Select (GE Healthcare). Band intensity was quantified with QuantityOne software (BioRad).

\section{Additional files}

Additional file 1: Cytoscheletal related genes in daDREAM hippocampus. qPCR analysis. (DOC $84 \mathrm{~kb}$ )

Additional file 2: Table S1. Assays from Applied Biosystems used for quantitative real-time PCR. (DOC 29 kb)

\section{Competing interests}

The authors declare that they have no competing interests.

\section{Authors' contributions}

BM designed and supervised biochemical experiments, analyzed data and wrote the paper. AK performed labeling experiments, Sholl analysis and analyzed data. SK performed labeling experiments, Sholl analysis and analyzed data. PG performed real-time GPCR. XMD did mouse genotyping, tissue collection and RNA preparation. ARN analyzed data and wrote the paper. IJ analyzed data and wrote the paper. MZ analyzed data and wrote the paper. TVPB analyzed data and wrote the paper. JRN conceived the study, designed experiments, analyzed data and wrote the paper. JdF designed and supervised morphometric experiments, analyzed data and wrote the paper. All authors read and approved the final manuscript.

\section{Acknowledgements}

This work was funded by Instituto de Salud Carlos III/CIBERNED (to JRN, BM and JdF), Madrid Community/Neurodegmodels (to JRN), by Ministerio de Economia y Competitividad (grants SAF2010-21784 and SAF2014-53412-R, to JRN) and by Areces Foundation, the EU 6th Framework Program: Network of Excellence NeuroNE (JRN) and the ERA-NET Programs Neuron (JRN), grants from the Medical Research Council (TVPB) and by grants from the EJLB-CIHR Michael Smith Chair in Neurosciences and Mental Health, Canada Research Chair, Canadian Institute for Health Research operating grant (MOP-124807), NSERC Discovery Grant (RGPIN 402555) (to MZ). SK had a postdoctoral contract from the Ramón y Cajal Program of the Ministry of Science and Innovation.

\section{Author details}

'Spanish Network for Biomedical Research in Neurodegenerative Diseases, CIBERNED, Madrid, Spain. ${ }^{2}$ National Biotechnology Center. CSIC, Darwin, 3. E-28049, Madrid, Spain. ${ }^{3}$ Cajal Institute, CSIC Madrid, Av Dr. Arce,37 E-28006, Madrid, Spain. ${ }^{4}$ Biomedical Technology Center, Politecnica University Madrid, Madrid, Spain. ${ }^{5}$ Neuronal Networks Group, School of Clinical and Experimental Medicine, University of Birmingham, Birmingham, UK ${ }^{6}$ Department of Physiology, Faculty of Medicine, University of Toronto, 1 King's College Circle, Toronto, Ontario, Canada. ${ }^{7}$ Center for Neuron and Disease, Frontier Institute of Science and Technology, Xi'an Jiaotong University, Xi'an, China. ${ }^{8}$ MRC National Institutes for Medical Research, Mill Hill, London, UK. ${ }^{9}$ Present address: IkerBasque Basque Foundation for Science and BioCruces, Health Research Institute, Bizkaia, Spain.

Received: 29 October 2015 Accepted: 22 February 2016

Published online: 29 February 2016

\section{References}

1. Malenka RC. The role of postsynaptic calcium in the induction of long-term potentiation. Mol Neurobiol. 1991;5:289-95.
2. Bading H, Ginty DD, Greenberg ME. Regulation of gene expression in hippocampal neurons by distinct calcium signaling pathways. Science. 1993;260:181-6.

3. Dolmetsch RE, Lewis RS, Goodnow CC, Healy Jl. Differential activation of transcription factors induced by Ca2+ response amplitude and duration. Nature. 1997;386:855-8.

4. Li W, Llopis J, Whitney M, Zlokarnik G, Tsien RY. Cell-permeant caged InsP3 ester shows that $\mathrm{Ca} 2+$ spike frequency can optimize gene expression. Nature. 1998:392:936-41.

5. Hardingham GE, Chawla S, Johnson CM, Bading H. Distinct functions of nuclear and cytoplasmic calcium in the control of gene expression. Nature. 1997:385:260-5

6. Cavazzini M, Bliss T, Emptage N. Ca2+ and synaptic plasticity. Cell Calcium 2005;38:355-67.

7. Segal I, Korkotian I, Murphy DD. Dendritic spine formation and pruning: common cellular mechanisms? Trends Neurosci. 2000;23:53-7.

8. Murphy DD, Segal M. Morphological plasticity of dendritic spines in central neurons is mediated by activation of CAMP response element binding protein. Proc Natl Acad Sci U S A. 1997:94:1482-7.

9. Carrion AM, Link WA, Ledo F, Mellstrom B, Naranjo JR. DREAM is a Ca2 + -regulated transcriptional repressor. Nature. 1999:398:80-4.

10. An WF, Bowlby MR, Betty M, Cao J, Ling HP, Mendoza G, et al. Modulation of A-type potassium channels by a family of calcium sensors. Nature. 2000;403:553-6.

11. Mellstrom B, Naranjo JR. Ca2 +-dependent transcriptional repression and derepression: DREAM, a direct effector. Semin Cell Dev Biol. 2001;12:59-63.

12. Mellstrom B, Savignac M, Gomez-Villafuertes R, Naranjo JR. Ca2 + -operated transcriptional networks: molecular mechanisms and in vivo models. Physiol Rev. 2008:88:421-49.

13. Ledo F, Kremer L, Mellstrom B, Naranjo JR. Ca2 + -dependent block of CREB-CBP transcription by repressor DREAM. Embo J. 2002;21:4583-92.

14. Wu LJ, Mellstrom B, Wang H, Ren M, Domingo S, Kim SS, et al. DREAM (Downstream Regulatory Element Antagonist Modulator) contributes to synaptic depression and contextual fear memory. Mol Brain. 2010;3:3.

15. Zhang Y, Su P, Liang P, Liu T, Liu X, Liu XY, et al. The DREAM protein negatively regulates the NMDA receptor through interaction with the NR1 subunit. J Neurosci. 2010;30:7575-86.

16. Mellstrom B, Sahun I, Ruiz-Nuno A, Murtra P, Gomez-Villafuertes R, Savignac $M$, et al. DREAM controls the on/off switch of specific activity-dependent transcription pathways. Mol Cell Biol. 2014;34:877-87.

17. Matus A. Actin-based plasticity in dendritic spines. Science. 2000;290:754-8.

18. Segal M. Dendritic spines: elementary structural units of neuronal plasticity. Prog Brain Res. 2002;138:53-9.

19. Yuste R, Bonhoeffer T. Morphological changes in dendritic spines associated with long-term synaptic plasticity. Annu Rev Neurosci. 2001;24:1071-89.

20. Guzowski JF, Lyford GL, Stevenson GD, Houston FP, McGaugh JL, Worley PF, et al. Inhibition of activity-dependent arc protein expression in the rat hippocampus impairs the maintenance of long-term potentiation and the consolidation of long-term memory. J Neurosci. 2000;20:3993-4001.

21. Tzingounis AV, Nicoll RA. Arc/Arg3.1: linking gene expression to synaptic plasticity and memory. Neuron. 2006;52:403-7.

22. Korb E, Finkbeiner S. Arc in synaptic plasticity: from gene to behavior. Trends Neurosci. 2011;34:591-8.

23. Forscher P. Calcium and polyphosphoinositide control of cytoskeletal dynamics. Trends Neurosci. 1989;12:468-74.

24. Naranjo JR, Mellstrom B. Ca2 + -dependent transcriptional control of Ca2+ homeostasis. J Biol Chem. 2012;287:31674-80.

25. Naranjo JR, Zhang H, Villar D, Gonzalez P, Dopazo XM, Moron-Oset J, et al. Activating transcription factor 6 derepression mediates neuroprotection in Huntington disease. J Clin Invest. 2016;126:627-38.

26. Cheng HY, Pitcher GM, Laviolette SR, Whishaw IQ, Tong Kl, Kockeritz LK, et al. DREAM is a critical transcriptional repressor for pain modulation. Cell. 2002:108:31-43.

27. Link WA, Ledo F, Torres B, Palczewska M, Madsen TM, Savignac M, et al. Day-night changes in downstream regulatory element antagonist modulator/potassium channel interacting protein activity contribute to circadian gene expression in pineal gland. J Neurosci. 2004:24:5346-55.

28. Mellstrom B, Torres B, Link WA, Naranjo JR. The BDNF gene: exemplifying complexity in $\mathrm{Ca} 2+-$ dependent gene expression. Crit Rev Neurobiol. 2004; 16:43-9. 
29. Gomez-Villafuertes R, Torres B, Barrio J, Savignac M, Gabellini N, Rizzato F, et al. Downstream regulatory element antagonist modulator regulates $\mathrm{Ca} 2+$ homeostasis and viability in cerebellar neurons. J Neurosci. 2005;25:10822-30.

30. Rivera-Arconada I, Benedet T, Roza C, Torres B, Barrio J, Krzyzanowska A, et al. DREAM regulates BDNF-dependent spinal sensitization. Mol Pain. 2010;6:95.

31. Star EN, Kwiatkowski DJ, Murthy VN. Rapid turnover of actin in dendritic spines and its regulation by activity. Nat Neurosci. 2002;5:239-46.

32. Simon-Areces J, Dopazo A, Dettenhofer M, Rodriguez-Tebar A, GarciaSegura LM, Arevalo MA. Formin1 mediates the induction of dendritogenesis and synaptogenesis by neurogenin3 in mouse hippocampal neurons. PLoS One. 2011;6:e21825.

33. Plath N, Ohana O, Dammermann B, Errington ML, Schmitz D, Gross C, et al. Arc/Arg3.1 is essential for the consolidation of synaptic plasticity and memories. Neuron. 2006:52:437-44.

34. Waung MW, Pfeiffer BE, Nosyreva ED, Ronesi JA, Huber KM. Rapid translation of Arc/Arg3.1 selectively mediates mGluR-dependent LTD through persistent increases in AMPAR endocytosis rate. Neuron. 2008;59:84-97.

35. Onoue K, Nakayama D, Ikegaya Y, Matsuki N, Nomura H. Fear extinction requires Arc/Arg3.1 expression in the basolateral amygdala. Mol Brain. 2014;7:30.

36. Peebles CL, Yoo J, Thwin MT, Palop JJ, Noebels JL, Finkbeiner S. Arc regulates spine morphology and maintains network stability in vivo. Proc Natl Acad Sci U S A. 2010;107:18173-8.

37. Arai A, Black J, Lynch G. Origins of the variations in long-term potentiation between synapses in the basal versus apical dendrites of hippocampal neurons. Hippocampus. 1994;4:1-9.

38. Fan W. Group I metabotropic glutamate receptors modulate late phase long-term potentiation in hippocampal CA1 pyramidal neurons: comparison of apical and basal dendrites. Neurosci Lett. 2013;553:132-7.

39. Kramar EA, Lynch G. Developmental and regional differences in the consolidation of long-term potentiation. Neuroscience. 2003;118:387-98.

40. Navakkode S, Sajikumar S, Korte M, Soong TW. Dopamine induces LTP differentially in apical and basal dendrites through BDNF and voltagedependent calcium channels. Learn Mem. 2012;19:294-9.

41. Sajikumar S, Navakkode S, Frey JU. Identification of compartment- and process-specific molecules required for "synaptic tagging" during long-term potentiation and long-term depression in hippocampal CA1. J Neurosci. 2007; $27: 5068-80$

42. Simon-Areces J, Membrive G, Garcia-Fernandez C, Garcia-Segura LM, Arevalo MA. Neurogenin 3 cellular and subcellular localization in the developing and adult hippocampus. J Comp Neurol. 2010;518:1814-24.

43. Zhou Q, Homma KJ, Poo MM. Shrinkage of dendritic spines associated with long-term depression of hippocampal synapses. Neuron. 2004:44:749-57.

44. Pintchovski SA, Peebles $\mathrm{CL}$, Kim HJ, Verdin E, Finkbeiner S. The serum response factor and a putative novel transcription factor regulate expression of the immediate-early gene Arc/Arg3.1 in neurons. J Neurosci. 2009;29:1525-37.

45. Kawashima T, Okuno H, Nonaka M, Adachi-Morishima A, Kyo N, Okamura M, et al. Synaptic activity-responsive element in the Arc/Arg3.1 promoter essential for synapse-to-nucleus signaling in activated neurons. Proc Natl Acad Sci U S A. 2009;106:316-21.

46. Smith-Hicks C, Xiao B, Deng R, Ji Y, Zhao X, Shepherd JD, et al. SRF binding to SRE 6.9 in the Arc promoter is essential for LTD in cultured Purkinje cells. Nat Neurosci. 2010;13:1082-9.

47. Sotiropoulos A, Gineitis D, Copeland J, Treisman R. Signal-regulated activation of serum response factor is mediated by changes in actin dynamics. Cell. 1999;98:159-69.

48. Tominaga T, Sahai E, Chardin P, McCormick F, Courtneidge SA, Alberts AS. Diaphanous-related formins bridge Rho GTPase and Src tyrosine kinase signaling. Mol Cell. 2000;5:13-25.

49. Young KG, Copeland JW. Formins in cell signaling. Biochim Biophys Acta. 1803;2010:183-90

50. Koch C, Poggio T, Torre V. Retinal ganglion cells: a functional interpretation of dendritic morphology. Philos Trans R Soc Lond B Biol Sci. 1982;298:227-63.

51. London M, Hausser M. Dendritic computation. Annu Rev Neurosci. 2005;28:503-32.

52. Spruston N. Pyramidal neurons: dendritic structure and synaptic integration. Nat Rev Neurosci. 2008:9:206-21.

53. van Elburg RA, van Ooyen A. Impact of dendritic size and dendritic topology on burst firing in pyramidal cells. PLoS Comput Biol. 2010;6:e1000781.
54. Poirazi P, Mel BW. Impact of active dendrites and structural plasticity on the memory capacity of neural tissue. Neuron. 2001:29:779-96.

55. Elston GN. Cortex, cognition and the cell: new insights into the pyramidal neuron and prefrontal function. Cereb Cortex. 2003;13:1124-38.

56. DeFelipe J. The dendritic spine story: an intriguing process of discovery. Front Neuroanat. 2015:9:14

57. Lilliehook C, Bozdagi O, Yao J, Gomez-Ramirez M, Zaidi NF, Wasco W, et al. Altered Abeta formation and long-term potentiation in a calsenilin knockout. J Neurosci. 2003;23:9097-106.

58. Alexander JC, MCDermott CM, Tunur T, Rands V, Stelly C, Karhson D, et al. The role of calsenilin/DREAM/KChIP3 in contextual fear conditioning. Learn Mem. 2009;16:167-77.

59. Savignac M, Pintado B, Gutierrez-Adan A, Palczewska M, Mellstrom B, Naranjo JR. Transcriptional repressor DREAM regulates T-lymphocyte proliferation and cytokine gene expression. Embo J. 2005;24:3555-64.

60. Kuo HC, Cheng CF, Clark RB, Lin JJ, Lin JL, Hoshijima M, et al. A defect in the KV channel-interacting protein 2 (KChIP2) gene leads to a complete loss of I(to) and confers susceptibility to ventricular tachycardia. Cell. 2001;107:801-13.

61. Xiong H, Xia K, Li B, Zhao G, Zhang Z. KChIP1: a potential modulator to GABAergic system. Acta Biochim Biophys Sin (Shanghai). 2009;41:295-300.

62. Buhl EH, Schlote W. Intracellular lucifer yellow staining and electron microscopy of neurones in slices of fixed epitumourous human cortical tissue. Acta Neuropathol. 1987;75:140-6.

63. Einstein G. Intracellular injection of lucifer yellow into cortical neurons in lightly fixed sections and its application to human autopsy material. J Neurosci Methods. 1988;26:95-103.

64. Elston GN, Pow DV, Calford MB. Neuronal composition and morphology in layer IV of two vibrissal barrel subfields of rat cortex. Cereb Cortex. 1997;7:422-31.

65. Hof PR. Comparative cytoarchitectonic atlas of the C57BL/6 and 129/Sv mouse brains. 2005

66. Lacaille JC, Mueller AL, Kunkel DD, Schwartzkroin PA. Local circuit interactions between oriens/alveus interneurons and CA1 pyramidal cells in hippocampal slices: electrophysiology and morphology. J Neurosci. 1987;7:1979-93.

67. Sholl DA. Dendritic organization in the neurons of the visual and motor cortices of the cat. J Anat. 1953:87:387-406.

68. Elston GN. Interlaminar differences in the pyramidal cell phenotype in cortical areas $7 \mathrm{~m}$ and STP (the superior temporal polysensory area) of the macaque monkey. Exp Brain Res. 2001;138:141-52.

69. Lein ES, Zhao X, Gage FH. Defining a molecular atlas of the hippocampus using DNA microarrays and high-throughput in situ hybridization. Jeurosci. 2004;24:3879-89.

70. Holmqvist MH, Cao J, Knoppers MH, Jurman ME, Distefano PS, Rhodes KJ, Xie Y, An WF. Kinetic modulation of Kv4-mediated A-current by arachidonic acid is dependent on potassium channel interacting proteins. J Neurosci. 2001;21(12):4154-61.

71. Ruiz-Gomez A, Mellström B, Tornero D, Morato E, Savignac M, Holguín H, Aurrekoetxea K, González P, González-García C, Ceña V, Mayor F Jr, Naranjo JR. G protein-coupled receptor kinase 2-mediated phosphorylation of downstream regulatory element antagonist modulator regulates membrane trafficking of Kv4.2 potassium channel. J Biol Chem. 2007;282(2):1205-15.

72. Lilliehook C, Chan S, Choi EK, Zaidi NF, Wasco W, Mattson MP, Buxbaum JD. Calsenilin enhances apoptosis by altering endoplasmic reticulum calcium signaling. Mol Cell Neurosci. 2002;19(4):552-9.

73. Ronkainen JJ, Hänninen SL, Korhonen T, Koivumäki JT, Skoumal R, Rautio S, Ronkainen VP, Tavi P. Ca2+-calmodulin-dependent protein kinase II represses cardiac transcription of the L-type calcium channel alpha(1C)subunit gene (Cacna1c) by DREAM translocation. J Physiol. 2011;589(Pt 11): 2669-86.

74. Thomsen MB, Wang C, Ozgen N, Wang HG, Rosen MR, Pitt GS. Accessory subunit KChIP2 modulates the cardiac L-type calcium current. Circ Res. 2009;104(12):1382-9.

75. Anderson D, Mehaffey WH, Iftinca M, Rehak R, Engbers JD, Hameed S, Zamponi GW, Turner RW. Regulation of neuronal activity by Cav3-Kv4 channel signaling complexes. Nat Neurosci. 2010;13(3):333-7.

76. Hu HJ, Carrasquillo Y, Karim F, Jung WE, Nerbonne JM, Schwarz TL, Gereau RW 4th. The kv4.2 potassium channel subunit is required for pain plasticity. Neuron. 2006;50(1):89-100 